\title{
Simple no-scale model of modulus fixing and inflation
}

\author{
John Ellis, ${ }^{1,2,3}$ Malcolm Fairbairn, ${ }^{1}$ Antonio Enea Romano, ${ }^{1,4}$ and Óscar Zapata ${ }^{4}$ \\ ${ }^{1}$ Theoretical Particle Physics and Cosmology Group, Department of Physics, \\ King's College London, London WC2R 2LS, United Kingdom \\ ${ }^{2}$ National Institute of Chemical Physics \& Biophysics, Rävala 10, 10143 Tallinn, Estonia \\ ${ }^{3}$ Theoretical Physics Department, CERN, CH-1211 Geneva 23, Switzerland \\ ${ }^{4}$ Instituto de Física, Universidad de Antioquia, A. A. 1226 Medellín, Colombia
}

(Received 26 June 2018; published 14 November 2018)

\begin{abstract}
We construct a no-scale model of inflation with a single modulus whose real and imaginary parts are fixed by simple power-law corrections to the no-scale Kähler potential. Assuming an uplift of the minimum of the effective potential, the model yields a suitable number of $e$-folds of expansion and values of the tilt in the scalar cosmological density perturbations and of the ratio of tensor and scalar perturbations that are compatible with measurements of the cosmic microwave background radiation.
\end{abstract}

DOI: 10.1103/PhysRevD.98.103514

\section{INTRODUCTION}

Cosmological inflation [1-4] provides one of the most promising arenas for probing physics close to the Planck scale, potentially even providing a window onto string theory. The effective energy scale during inflation may well be within a few orders of magnitude of the string scale, and in a wide class of inflationary models the excursion in the effective inflaton field is trans-Planckian. It is therefore natural to use string theory as an inspiration for the construction of such models, or at least to constrain the model-builders' imaginations [5].

Consistent string models generally incorporate supersymmetry, and there are many practical reasons for supposing that supersymmetry may become apparent at some energy scale below that of inflation [6]. These considerations motivate the construction of supersymmetric models of inflation, which also offer advantages in rendering more natural the apparent hierarchy between the Planck scale and the energy scale during inflation [7]. Since inflation is a cosmological scenario that necessarily involves gravity, the most plausible supersymmetric framework for constructing models of inflation is actually supergravity [8]. Within this general framework, no-scale supergravity [9-12] stands out [13-18], since at the classical level it has a positivesemidefinite potential with flat directions that do not restrict field excursions [9]. Moreover, it emerges as the form of

Published by the American Physical Society under the terms of the Creative Commons Attribution 4.0 International license. Further distribution of this work must maintain attribution to the author(s) and the published article's title, journal citation, and DOI. Funded by SCOAP. low-energy field theory derived from compactifications of string theory [19].

The simplest no-scale supergravity model has a single complex field $T$ that parametrizes a noncompact $\mathrm{SU}(1,1) /$ $\mathrm{U}(1)$ coset manifold with a Kähler potential $K=$ $-3 \ln \left(T+T^{*}\right)[9,10]$, and would correspond to the volume modulus in a string compactification [19]. It is a muchdebated, very general and open, question how the values of the real and imaginary components of this and other compactification moduli could be fixed dynamically in the low-energy physical vacuum $[20,21] .{ }^{1}$ It is natural also to ask whether (some component) of the $T$ field could serve as the inflaton, and how this could be combined with whatever mechanism that fixes dynamically the real and imaginary components of $T$.

In this paper, we explore a possible common solution to these problems that postulates power-law modifications of the leading-order Kähler potential of the form $\Delta K=c_{n} /\left(T+T^{*}\right)^{n}+d_{m} /\left(T-T^{*}\right)^{m}$, the first of which is rooted in our understanding of perturbative corrections to string compactifications $[23,24]$. We show that, for suitable values of the powers $n, m$ and the correction parameters $c_{n}$, $d_{m}$, there is a unique minimum of the effective potential $V<0$ with fixed values of both the real and imaginary parts of $T$. We recognize that the solution of the cosmological constant problem is unknown, and is in particular an unsolved problem in string theory [25] that must await a more complete understanding of quantum gravity. In the absence of a solution to this problem, we assume here that some unspecified uplifting mechanism, e.g., in the sector of

\footnotetext{
${ }^{1}$ An alternative would be to consider a scenario in which the quantum degree of freedom corresponding to $T-T^{*}$ is an (almost) massless axionlike particle [22].
} 
the theory that fixes the string coupling, raises the minimum of the effective potential to $V \simeq 0$. We then explore the possibility of successful inflation with the resulting positive semidefinite potential $V(T)$, assuming that this is not modified significantly by the unspecified uplifting mechanism. We find regions of initial conditions for the real and imaginary parts of $T$ that yield a number of $e$-folds $N_{*}$ and values of the scalar tilt parameter $n_{s}$ and the ratio of tensor to scalar perturbations $r$ that are highly compatible with the available data on the cosmic microwave background (CMB) data: $N_{*} \sim 55, n_{s}=0.967$ and $r \sim 0.0007$ [26]. This model therefore provides a successful scenario for inflation in the context of a minimal string-inspired noscale supergravity model.

\section{THE EFFECTIVE POTENTIAL AND MODULUS FIXING}

We recall that an $\mathcal{N}=1$ supergravity theory is specified [27] by a Hermitian Kähler function $K$ and a holomorphic superpotential $W$ via the combination

$$
G \equiv K+\ln W+\ln W^{*} .
$$

The Kähler function specifies the kinetic terms for the scalar fields

$$
K_{i}^{j^{*}} \equiv \frac{\partial^{2} K}{\partial \phi^{i} \partial \phi_{j}^{*}},
$$

where $K_{i}^{j^{*}} \equiv \partial^{2} K / \partial \phi^{i} \partial \phi_{j}^{*}$ is the Kähler metric, and the effective potential is

$$
V=e^{G}\left[\frac{\partial G}{\partial \phi^{i}} K_{j^{*}}^{i} \frac{\partial G}{\partial \phi_{j}^{*}}-3\right]+\text { possible } D \text {-terms }
$$

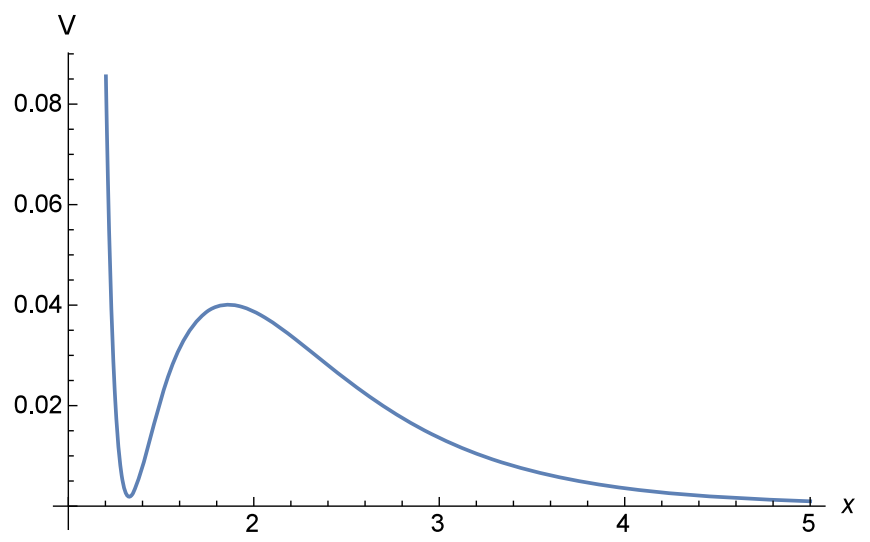

FIG. 1. The effective potential (6) as a function of $x=T+T^{*}$ for $n=4, \lambda=-1, c_{n}=3$ and $\beta=1$.

and $K_{j^{*}}^{i}$ is the inverse of the Kähler metric. In the following, we study the simplest possible $\mathcal{N}=1$ supergravity model with a single complex scalar field $T$, and an exponential superpotential for $T: W(T)=\beta e^{\lambda T}$. As mentioned in the Introduction, the minimal $\mathcal{N}=1$ no-scale supergravity model has a Kähler potential $K=-3 \ln \left(T+T^{*}\right)[9,10]$. We consider initially a Kähler potential with a correction of the form ${ }^{2}$ :

$$
K=-3 \ln \left(T+T^{*}\right)+\frac{c_{n}}{\left(T+T^{*}\right)^{n}},
$$

which yields a Kähler potential

$$
K_{T}^{T^{*}}=\frac{\left(T+T^{*}\right)^{n+2}}{c_{n} n(n+1)+3\left(T+T^{*}\right)^{n}} .
$$

In the following, we denote the real part of $T$ by $x$ and the imaginary part by $y: T=x+i y$, and define $g(x) \equiv K_{T}^{T^{*}}$. The resulting effective potential is

$$
V(x)=\frac{\beta^{2} x^{-n-3} e^{c_{n} x^{-n}-\lambda x}\left(c_{n}^{2} n^{2}-c_{n} n x^{n}(3 n-2 \lambda x-3)+\lambda x^{2 n+1}(\lambda x+6)\right)}{c_{n} n(n+1)+3 x^{n}} .
$$

The effective potential $V(x)$ has a local minimum at a nonzero value of $x$ when $n \geq 2$, as illustrated in Fig. 1 for the specific choices $n=4, \lambda=-1, c_{n}=3$ and $\beta=1$. $^{3}$

This is not the first example of stabilization of the real part of $T$ (see, e.g., [21]), but stabilization of the imaginary part has proved more elusive (see, however, [28]). In particular, the effective potential (6) is

\footnotetext{
${ }^{2}$ This form is inspired by the form of effective field theory found in [23] in describing $(2,2)$ vacua of the heterotic string.

${ }^{3}$ The other plots of the effective potential shown below are also for $\beta=1$, but similar results are obtained with other values of $\beta$.
}

independent of $y$. In order to explore how $y$ may also be stabilized, we next consider adding instead to the noscale Kähler potential a dependence on the imaginary part of the modulus $T$ that is also of power-law form, though not sharing its motivation from calculations of $\alpha^{\prime}$ corrections in string theory [23,24]:

$$
K=-3 \ln \left(T+T^{*}\right)+\frac{d_{m}}{\left|T-T^{*}\right|^{m}}
$$

In this case the effective potential takes the following form for $x, y>0$ : 

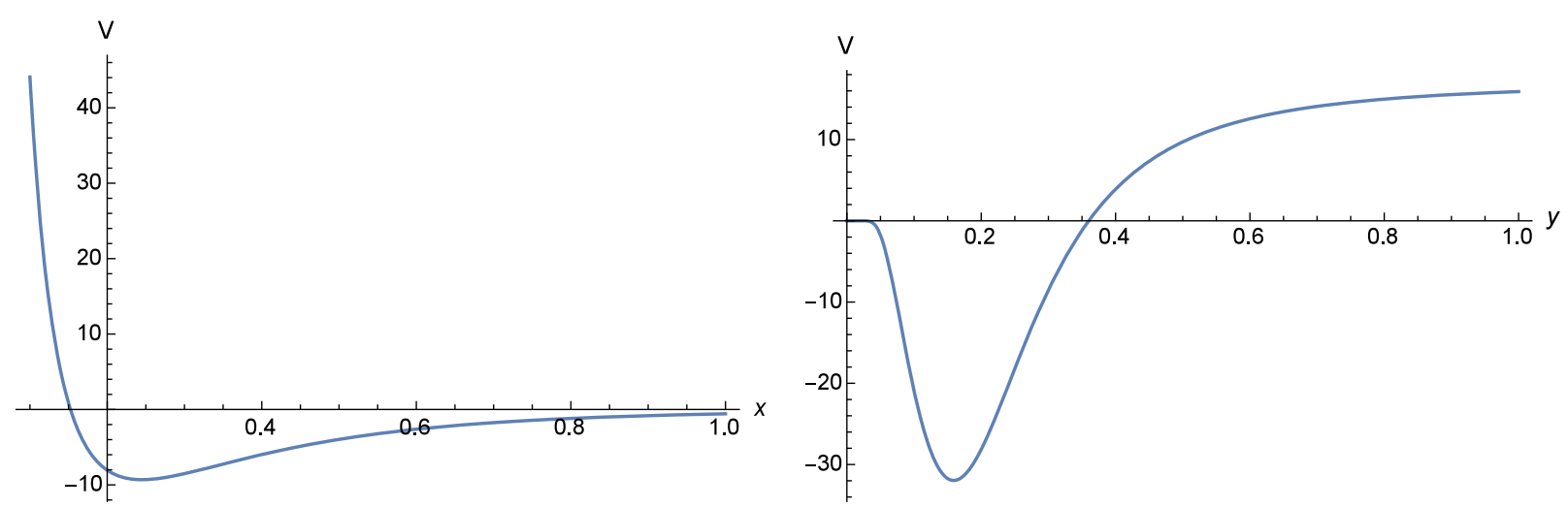

FIG. 2. The effective potential obtained from (7) for $d_{m}=-0.05, m=3 / 2, \lambda=-1$ is plotted in the left panel as a function of $x$ for fixed $y=0.3$, and in the right panel as a function of $y$ for fixed $x=0.3$. Both plots are for $\beta=1$.

$$
\begin{aligned}
V= & \frac{\beta^{2}}{x^{3}} \exp \left[d_{m} y^{-m}+\lambda x\right]\left[-3+\frac{1}{\left(-d_{m} m(m+1) y^{-m-2}+\frac{3}{x^{2}}\right)}\right. \\
& \left.\times\left(-d_{m} m y^{-m-1}+\lambda-\frac{3}{x}\right)\left(d_{m} m y^{-m-1}+\lambda \frac{3}{x}\right)\right]
\end{aligned}
$$

Figure 2 displays two slices through the effective potential (8) for $c_{n}=0, d_{m}=-0.05, m=3 / 2$ and $\lambda=-1$. In the left panel we show an $x$ slice for fixed $y=0.3$, and in the right panel we show a $y$ slice for fixed $x=0.3$. We see that in both slices there is a nontrivial minimum. We have also explored whether this example is suitable for inflation, but found that this was not the case, and so do not consider further the $c_{n}=0$ option.

We have instead considered adding both the $T+T^{*}$ dependent term in (4) and the $T-T^{*}$-dependent term in (7) simultaneously to the no-scale Kähler potential:

$$
K=-3 \ln \left(T+T^{*}\right)+\frac{c_{n}}{\left|T+T^{*}\right|^{n}}+\frac{d_{m}}{\left|T-T^{*}\right|^{m}}
$$

In this case the effective potential takes the following form for $x, y>0$ :

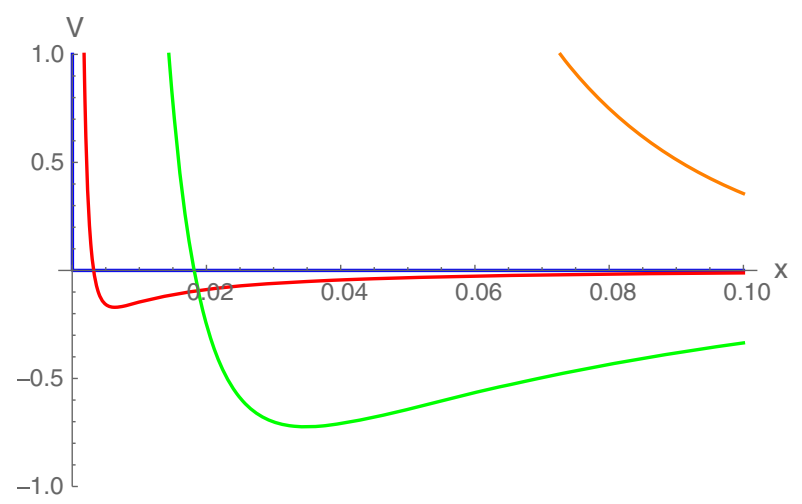

$$
\begin{aligned}
V= & \frac{\beta^{2}}{x^{3}} \exp \left[c_{n} x^{-n}+d_{m} y^{-m}+\lambda x\right] \\
& \times\left[-3+\frac{1}{c_{n} n(n+1) x^{-n-2}-d_{m} m(m+1) y^{-m-2}+\frac{3}{x^{2}}}\right. \\
& \times\left(-c_{n} n x^{-n-1}-d_{m} m y^{-m-1}+\lambda-\frac{3}{x}\right) \\
& \left.\times\left(-c_{n} n x^{-n-1}+d_{m} m y^{-m-1}+\lambda \frac{3}{x}\right)\right] .
\end{aligned}
$$

Fig. 3 shows slices through the effective potential (10) for the choices $n=-2, m=3 / 2, \lambda=-1, c_{n}=-5.9$, $d_{m}=-4.44$. The left panel shows the $x$ dependence of the potential for several fixed values of $y$ and the right panel shows the $y$ dependence for several fixed values of $x$. We see that the real component $x$ of the modulus $T$ is fixed at a nonzero value for the values $y=\{0.5,0.7\}$, and that the imaginary component $y$ is fixed at a nonzero value for the values $x=\{0.04,0.06,0.08,0.1\}$.

Figure 4 shows a three-dimensional image of the potential (10) for the same parameter choices $n=-2$, $m=3 / 2, \lambda=-1, c_{n}=-5.9, d_{m}=-4.44$ used in Fig. 3 .

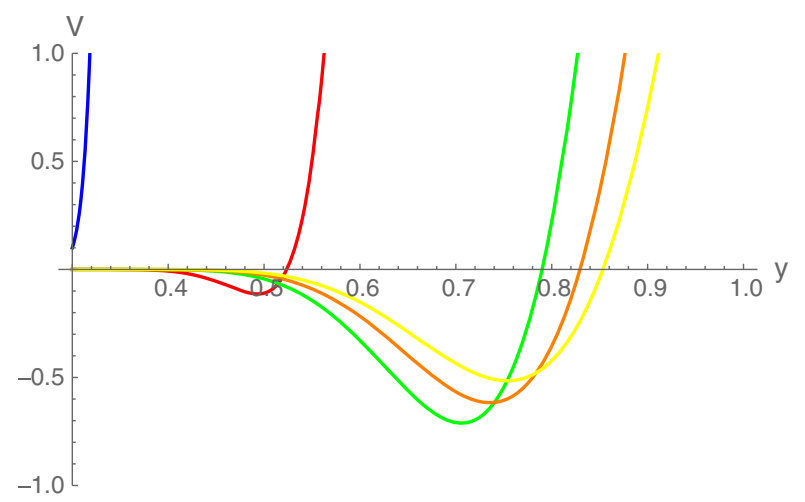

FIG. 3. The effective potential (10) for $n=-2, m=3 / 2, \lambda=-1, c_{n}=-5.9, d_{m}=-4.44$ is plotted in the left panel as a function of $x$, with the blue, red, green, and orange lines corresponding to $y=\{0.3,0.5,0.7,0.9\}$, and in the right panel as a function of $y$ with the blue, red, green, orange and yellow lines corresponding to $x=\{0.004,0.04,0.06,0.08,0.1\}$. Both plots are for $\beta=1$. 


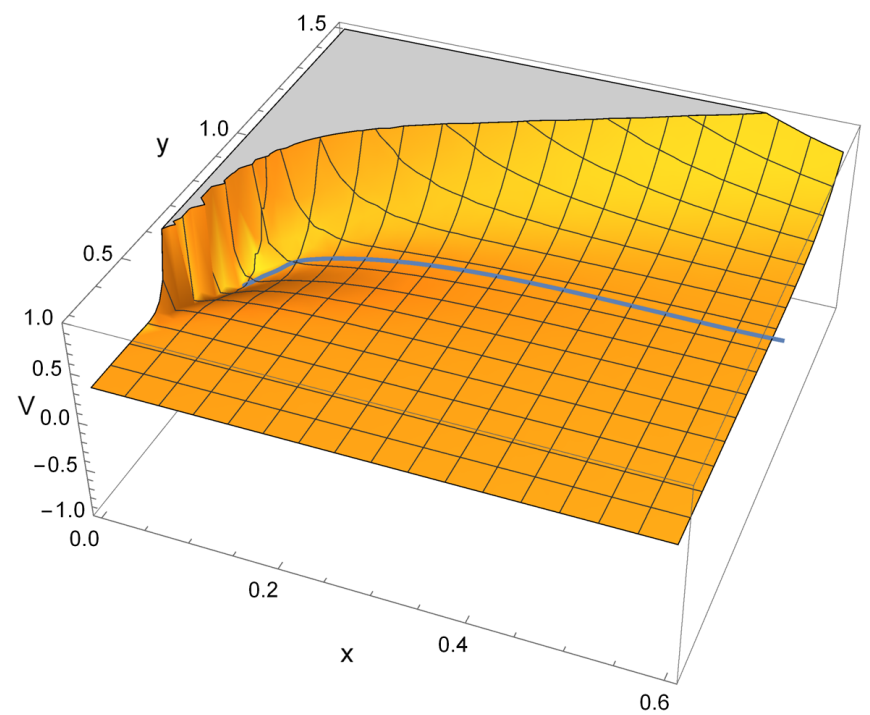

FIG. 4. The effective potential (10) is plotted as a function of $x$ and $y$ for $n=-2, m=3 / 2, \lambda=-1, c_{n}=-4.44, d_{m}=-5.9$ and $\beta=1$. A global minimum with $x, y \neq 0$ is clearly present. Also shown as a blue line is a possible field trajectory.

This confirms that there is indeed a global minimum of the potential with $x, y \neq 0$. Thus, the ansatz (9) achieves the goal of fixing both the components of the modulus field $T$.

\section{A REALIZATION OF INFLATION}

The effective potential shown in Fig. 4 exhibits an extended flat region in addition to the global minimum, and we now study whether there are field trajectories ending in the minimum that are suitable for cosmological inflation. In order to check this, we need to solve the equations of motion for the modulus field components $x, y$ in an expanding Universe described by a FriedmanRobertson-Walker (FRW) metric

$$
d s^{2}=-d t^{2}+a(t)^{2} d \vec{x}^{2},
$$

corresponding to the action

$$
S=\int \sqrt{-g}\left[K_{T T^{*}} \partial_{\mu} T \partial^{\mu} T^{*}-V\right] d^{4} x .
$$

in curved space. Assuming a homogeneous FRW background, only the time derivative survives in the kinetic term and we obtain the following effective Lagrangian

$$
L=a(t)^{3}\left[K_{T T^{*}}(x, y)\left(\dot{x}^{2}+\dot{y}^{2}\right)-V(x, y)\right],
$$

where

$$
\begin{aligned}
K_{T T^{*}}= & \left(c_{n} n(n+1) x^{-n-2}-d_{m} m(m+1) y^{-m-2}\right. \\
& \left.+e_{p} p(p+1) x^{-p-2}+\frac{3}{x^{2}}\right) .
\end{aligned}
$$

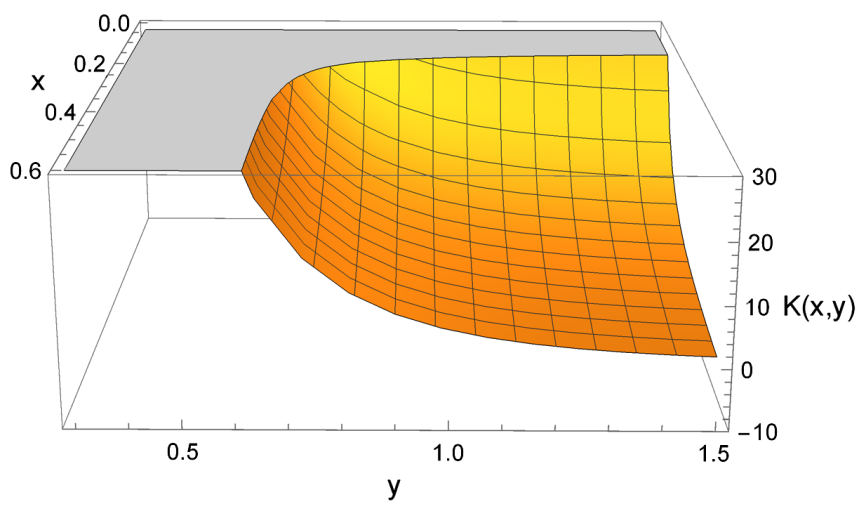

FIG. 5. The function $K_{T T^{*}}(x, y)$ is plotted as a function of $x$ and $y$ for $n=-2, m=3 / 2, \lambda=-1, c_{n}=-4.44, d_{m}=-5.9$. At small $x, y$ it becomes large enough to make the kinetic term dominant respect to the potential and impede inflation, despite the flatness of the potential.

In this case the energy-momentum tensor obtained from the effective Lagrangian in Eq. (13) corresponds to a perfect fluid with $P=K_{T T^{*}}(x, y)\left(\dot{x}^{2}+\dot{y}^{2}\right)-V(x, y)$, $\rho=K_{T T^{*}}(x, y)\left(\dot{x}^{2}+\dot{y}^{2}\right)+V(x, y)$. Due to the nontrivial kinetic term, the flatness of the potential is not a sufficient condition for the acceleration of the scale factor to be positive. According to Friedmann's equations, $\frac{\ddot{a}}{a} \propto-(\rho+3 P)$, so the general condition for accelerated expansion is $P<-\rho / 3$.

Figure 5 shows a plot of $K_{T T^{*}}(x, y)$, which becomes relatively large for $y<0.7$. In such regions the flatness of the potential is not enough for inflationary expansion, because the kinetic term can dominate the potential despite its flatness, leading to non-negative pressure. Numerical solutions of the background equations confirm this analysis, but there is still a large region of the field space where inflation is possible, as we now show. No fine tuning of the initial conditions is required to get inflationary trajectories in the region where the potential is flat and $K_{T T^{*}}(x, y)$ is not too large.

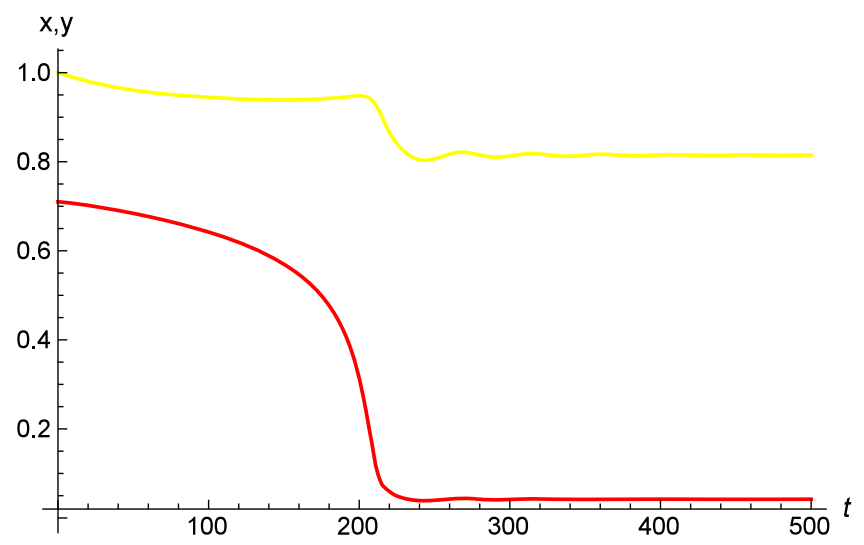

FIG. 6. The field components $x$ and $y$ are plotted in red and yellow, respectively, as functions of the time $t$ along the field trajectory shown in Fig. 4. 

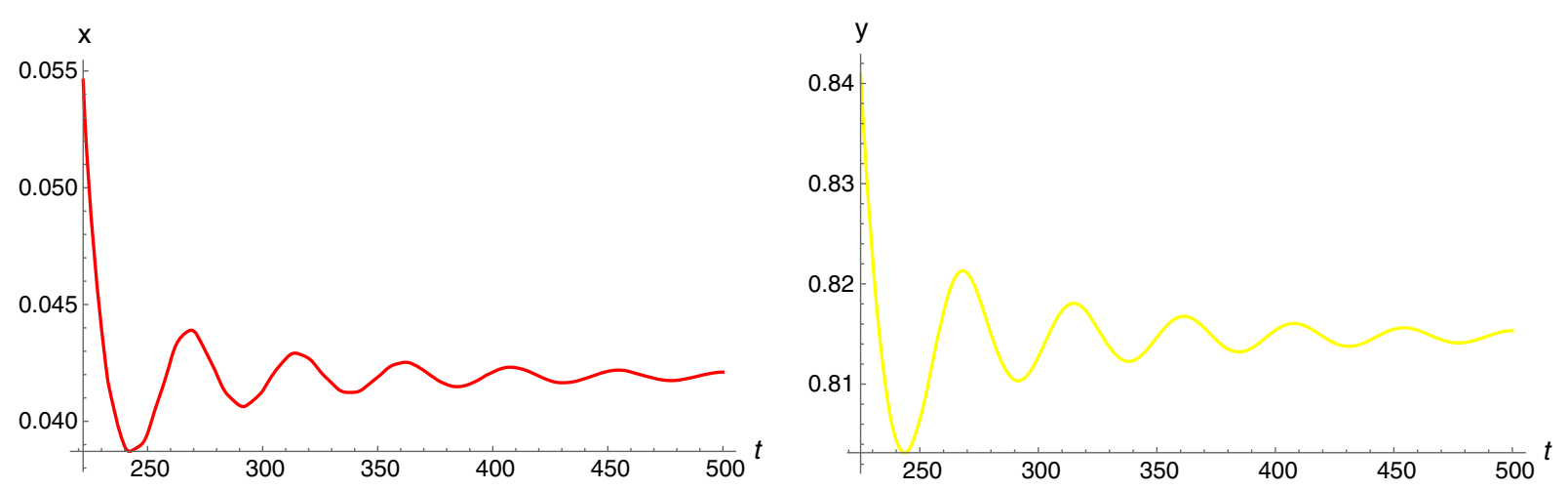

FIG. 7. The field components $x$ and $y$ are plotted as functions of the time $t$ along the field trajectory shown in Fig. 4, during the oscillatory phase at the end of inflation, as they are approaching the minimum of the potential.

The Lagrange equations for the fields $x$ and $y$ and the Einstein equations for the scale factor $a(t)$ yield the following system of differential equations that describe completely the classical background field evolution:

$$
\begin{gathered}
\frac{d}{d t}\left(\frac{\partial L}{\partial \dot{x}}\right)-\frac{\partial L}{\partial x}=0, \\
\frac{d}{d t}\left(\frac{\partial L}{\partial \dot{y}}\right)-\frac{\partial L}{\partial y}=0, \\
\left.H^{2}=\frac{1}{3}\left[K_{T T^{*}}(x, y)\left(\dot{x}^{2}+\dot{y}^{2}\right)+V(x, y)\right)\right] .
\end{gathered}
$$

We solve numerically and exactly this system of three differential equations for the background evolutions of the functions $x(t), y(t), a(t)$, making no approximation of any kind.

A representative solution of these equations of motions is shown in Fig. 4, as a blue line that starts at $\{x, y\}=\{0.71,1\}$ and terminates at the global minimum. Figures 6-7 display the evolutions along the field trajectory shown in Fig. 4 of the real and imaginary components $\{x, y\}$ of $T$ as functions of time, as red and yellow lines, respectively. We see that $x$ decreases smoothly for $t \lesssim 240$, after which its value evolves only slowly, exhibiting small oscillations. The value of $y$ changes by $\lesssim 10 \%$ for $t \lesssim 240$, after which it drops to an almost constant value that also exhibits small oscillations.

Integrating the background equations, we can compute the slow-roll parameters along the field trajectories, where we adopt the following definitions [29]:

$$
\begin{gathered}
\epsilon_{1}=-\dot{H} / H^{2}, \\
\epsilon_{i+1} \equiv \dot{\epsilon}_{i} /\left(H \epsilon_{i}\right), \\
n_{s}-1=-2 \epsilon_{1}-\epsilon_{2}-2 \epsilon_{1}^{2}, \\
r=16 \epsilon_{1} .
\end{gathered}
$$

Figure 8 displays the evolutions along the field trajectory shown in Fig. 4 of the Hubble parameter $H$ (green line), the slow-roll parameter $\epsilon_{1}$ (blue line), and the number of $e$-folds of expansion $N$ (black line, rescaled by a reference value of 70). We see that the Hubble parameter varies only slowly until a time $t \simeq 200$, falling to much smaller values when $t \gtrsim 240$. Correspondingly, the number of $e$-folds $N$ increases nearly linearly until $t \sim 200$, after which it is nearly constant. The value of $\epsilon$ is small until a similar value of $t$, after which it enters a period of damped oscillations with amplitudes that are initially $\mathcal{O}(1)$.

The initial conditions $x_{i}=0.71, y_{i}=1, \dot{y}_{i}=\dot{x}_{i}=0$ lead to the field trajectory shown in Fig. 4, which yields a number of $e$-folds $N \sim 55$, a scalar perturbation tilt $n_{s}=$ 0.967 and tensor-to-scalar perturbation ratio $r=0.00069$, which are compatible with the observational constraints [26]. Other choices of initial conditions also yield acceptable inflationary trajectories. For example, changing the initial value of $y$ to 0.9 but keeping the initial value of $x$ fixed yields $n_{s}=0.961$ and $r=0.00030$, whilst $y=1.1$

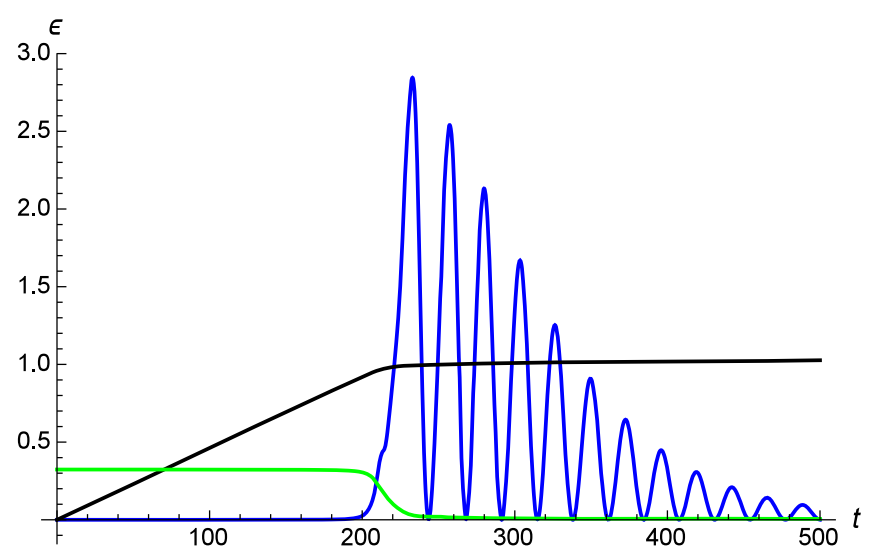

FIG. 8. The Hubble parameter $H$, the slow-roll parameter $\epsilon$ and the rescaled number of $e$-folds $N / 70$ are plotted as functions of time along the field trajectory shown in Fig. 4 as green, blue and black lines, respectively. 
yields $n_{s}=0.967$ and $r=0.00075$, also compatible with the observations.

As mentioned above, it is easy to find viable models with different values of $\beta$. For example, with $\beta=2$ the initial choices $x_{i}=0.68, y_{i}=0.9, \dot{y}_{i}=0.002, \dot{x}_{i}=0.0009$ yield successful inflation. On the other hand, as explained above, for $y<0.7$ the nontriviality of the kinetic term makes inflation impossible, despite the flatness of the effective potential. However, the region where inflation is possible is still quite large, and no fine-tuning is required for the initial conditions of successful inflation trajectories.

\section{CONCLUSIONS}

We have presented in this paper a simple scenario for fixing both components of the modulus $T$ in the minimal no-scale supergravity model with Kähler potential $K=$ $-3 \ln \left(T+T^{\dagger}\right)$, which is partly based upon theoretical calculations of corrections to this structure [23,24]. In addition to yielding an effective potential that possesses a well-defined, unique minimum, this simple model exhibits a plateau at larger values of the components of $T$. We have found examples of field trajectories starting from initial values in this plateau region that yield an acceptable number of $e$-folds of inflation and values of the CMB observables $n_{s}$ and $r$ that are compatible with observation [26].

One interesting direction for future research will be to map out more completely the parameter space of initial field values that are compatible with cosmological observations, another will be to reconcile it better with string considerations, and another will be to integrate this simple scenario into a framework with matter particles and a scenario for reheating that would enable the number of inflationary $e$-folds to be calculated. In this way, the simple model presented in this paper may serve as the kernel of a more complete cosmological model.

\section{ACKNOWLEDGMENTS}

J. E. thanks Ruben Minasian for useful discussions. We thank Joe Conlon for pointing out en error in the first version of this paper. The work of J.E. and M.F. was supported partly by the STFC Grant No. ST/P000258/1. The work of J. E. was also supported partly by the Estonian Research Council via a Mobilitas Pluss grant and that of M. F. was also supported by the European Research Council under the European Union's Horizon 2020 program (ERC Grant Agreement No. 648680 DARKHORIZONS). The work of A.E. R. was supported by the Dedicación Exclusiva and Sostenibilidad programs at the Universidad de Antioquia, as well as its CODI Projects No. 2015-4044, No. 2016-10945, and No. 2016-13222, and the Colciencias mobility program. The work of O.Z. was supported partly by Colciencias through Grants No. 111565842691 and No. 111577657253.
[1] A. A. Starobinsky, Phys. Lett. 91B, 99 (1980).

[2] A. H. Guth, Phys. Rev. D 23, 347 (1981).

[3] V. F. Mukhanov and G. V. Chibisov, Pis'ma Zh. Eksp. Teor. Fiz. 33, 549 (1981) [JETP Lett. 33, 532 (1981)].

[4] A. D. Linde, Phys. Lett. 108B, 389 (1982); A. Albrecht and P. J. Steinhardt, Phys. Rev. Lett. 48, 1220 (1982).

[5] S. Kachru, R. Kallosh, A. Linde, J. Maldacena, L. McAllister, and S. P. Trivedi, J. Cosmol. Astropart. Phys. 310 (2003) 013.

[6] L. Maiani, Proceedings of Summer School on Particle Physics, Gif-sur-Yvette, 1979 (IN2P3, Paris, 1980), p. 3; G. 't Hooft, in Recent Developments in Field Theories, edited by G. 't Hooft et al. (Plenum Press, New York, 1980); E. Witten, Nucl. Phys. B188, 513 (1981); R. K. Kaul, Phys. Lett. 109B, 19 (1982); J. R. Ellis, J. S. Hagelin, D. V. Nanopoulos, K. A. Olive, and M. Srednicki, Nucl. Phys. B238, 453 (1984); J. Ellis, S. Kelley, and D. V. Nanopoulos, Phys. Lett. B 260, 131 (1991); U. Amaldi, W. de Boer, and H. Furstenau, Phys. Lett. B 260, 447 (1991); P. Langacker and M. Luo, Phys. Rev. D 44, 817 (1991); C. Giunti, C. W. Kim, and U. W. Lee, Mod. Phys. Lett. A 06, 1745 (1991); J. R. Ellis, G. Ridolfi, and F. Zwirner, Phys. Lett. B 257, 83 (1991); H. E. Haber and R. Hempfling, Phys. Rev. Lett. 66, 1815 (1991); Y. Okada,
M. Yamaguchi, and T. Yanagida, Prog. Theor. Phys. 85, 1 (1991).

[7] J. R. Ellis, D. V. Nanopoulos, K. A. Olive, and K. Tamvakis, Phys. Lett. 118B, 335 (1982); Nucl. Phys. B221, 52 (1983); K. Nakayama and F. Takahashi, J. Cosmol. Astropart. Phys. 10 (2011) 033.

[8] D. V. Nanopoulos, K. A. Olive, M. Srednicki, and K. Tamvakis, Phys. Lett. 123B, 41 (1983); R. Holman, P. Ramond, and G. G. Ross, Phys. Lett. 137B, 343 (1984); A. B. Goncharov and A. D. Linde, Phys. Lett. 139B, 27 (1984).

[9] E. Cremmer, S. Ferrara, C. Kounnas, and D. V. Nanopoulos, Phys. Lett. 133B, 61 (1983); J. R. Ellis, A. B. Lahanas, D. V. Nanopoulos, and K. Tamvakis, Phys. Lett. 134B, 429 (1984).

[10] J. R. Ellis, C. Kounnas, and D. V. Nanopoulos, Nucl. Phys. B241, 406 (1984).

[11] J. R. Ellis, C. Kounnas, and D. V. Nanopoulos, Nucl. Phys. B247, 373 (1984).

[12] A. B. Lahanas and D. V. Nanopoulos, Phys. Rep. 145, 1 (1987).

[13] A.S. Goncharov and A. D. Linde, Classical Quantum Gravity 1, L75 (1984).

[14] C. Kounnas and M. Quiros, Phys. Lett. 151B, 189 (1985).

[15] J. R. Ellis, K. Enqvist, D. V. Nanopoulos, K. A. Olive, and M. Srednicki, Phys. Lett. 152B, 175 (1985); 156B, 452(E) (1985). 
[16] K. Enqvist, D. V. Nanopoulos, and M. Quiros, Phys. Lett. 159B, 249 (1985); P. Binétruy and M. K. Gaillard, Phys. Rev. D 34, 3069 (1986); H. Murayama, H. Suzuki, T. Yanagida, and J. Yokoyama, Phys. Rev. D 50, R2356 (1994); S. C. Davis and M. Postma, J. Cosmol. Astropart. Phys. 03 (2008) 015; S. Antusch, M. Bastero-Gil, K. Dutta, S. F. King, and P. M. Kostka, J. Cosmol. Astropart. Phys. 01 (2009) 040; S. Antusch, M. Bastero-Gil, K. Dutta, S. F. King, and P. M. Kostka, Phys. Lett. B 679, 428 (2009); R. Kallosh and A. Linde, J. Cosmol. Astropart. Phys. 11 (2010) 011; R. Kallosh, A. Linde, and T. Rube, Phys. Rev. D 83, 043507 (2011); S. Antusch, K. Dutta, J. Erdmenger, and S. Halter, J. High Energy Phys. 04 (2011) 065; R. Kallosh, A. Linde, K. A. Olive, and T. Rube, Phys. Rev. D 84, 083519 (2011); T. Li, Z. Li, and D. V. Nanopoulos, J. Cosmol. Astropart. Phys. 02 (2014) 028; W. Buchmuller, C. Wieck, and M. W. Winkler, Phys. Lett. B 736, 237 (2014).

[17] J. Ellis, D. V. Nanopoulos, and K. A. Olive, Phys. Rev. Lett. 111, 111301 (2013).

[18] J. Ellis, M. A. G. Garcia, D. V. Nanopoulos, and K. A. Olive, Classical Quantum Gravity 33, 094001 (2016).

[19] E. Witten, Phys. Lett. 155B, 151 (1985).

[20] S. Kachru, R. Kallosh, A. D. Linde, and S. P. Trivedi, Phys. Rev. D 68, 046005 (2003).
[21] J. R. Ellis, C. Kounnas, and D. V. Nanopoulos, Phys. Lett. 143B, 410 (1984); J. Ellis, D. V. Nanopoulos, and K. A. Olive, J. Cosmol. Astropart. Phys. 10 (2013) 009.

[22] D. J. E. Marsh, Phys. Rep. 643, 1 (2016).

[23] L. J. Dixon, V. Kaplunovsk, and J. Louis, Nucl. Phys. B329, 27 (1990).

[24] K. Becker, M. Becker, M. Haack, and J. Louis, J. High Energy Phys. 06 (2002) 060; R. Minasian, T. G. Pugh, and R. Savelli, J. High Energy Phys. 10 (2015) 050.

[25] U. H. Danielsson and T. Van Riet, arXiv:1804.01120.

[26] P. A. R. Ade et al. (Planck Collaboration), Astron. Astrophys. 594, A13 (2016); 594, A20 (2016).

[27] E. Cremmer, B. Julia, J. Scherk, S. Ferrara, L. Girardello, and P. van Nieuwenhuizen, Nucl. Phys. B147, 105 (1979); E. Cremmer, S. Ferrara, L. Girardello, and A. Van Proeyen, Nucl. Phys. B212, 413 (1983).

[28] J. Ellis, M. A. G. Garcia, D. V. Nanopoulos, and K. A. Olive, J. Cosmol. Astropart. Phys. 08 (2014) 044.

[29] See, e.g., D. H. Lyth and A. R. Liddle, The Primordial Density perturbation: Cosmology, Inflation and the Origin of Structure (Cambridge University Press, Cambridge, England, 2009). 